\title{
Technique for reloading over-the-scope clip using three needle holders
}

\section{George Tribonias, Ioannis Giotis, Maria Palatianou, Maria Tzouvala}

General Hospital of Nikaia-Piraeus "Agios Panteleimon”, Athens, Greece

We describe the case of a 64-year-old woman with subtotal gastrectomy due to gastric cancer. One month after the operation the patient presented abdominal pain, fever, and a palpable mass above the surgical incision. An abdominal abscess was detected and drained under computed tomographic guidance. The secretion was first purulent and finally bilious. Gastroscopy with fluoroscopy was performed and revealed leakage in the gastroenteric anastomosis. An over-the-scope clip (OTSC) was selected to stop the leakage by closing the defect [1]. At the beginning of the intervention, the clip was released because of an improper manipulation while it was being loaded onto the endoscope. We decided to attempt to reload the clip using the following process (Fig. 1): The clip was grasped by the edges of the lower side with 2 needle holders, while a third needle holder grasped the upper side in the middle. First, the lower side was fitted into the cap through the thread and held stable with the 2 needle holders. Then, we started to pull the upper side slowly, until the clip was reopened and embraced the cap. The OTSC was successfully reloaded and finally released, closing the defect with fluoroscopic confirmation (Fig. 2). This technique is unique in the literature and could possibly be used to deal not only with a "human error", but also the restricted availability of OTSC due to its high cost.

Department of Gastroenterology, General Hospital of Nikaia-Piraeus "Agios Panteleimon", Athens, Greece

\section{Conflict of Interest: None}

Correspondence to: George Tribonias MD, PhD, Department of Gastroenterology, General Hospital of Nikaia-Piraeus "Agios Panteleimon", Mantouvalou D. 3, 18454 Athens, Greece, e-mail: g.tribonias@gmail.com

Received 13 March 2020; accepted 18 March 2020; published online 10 May 2020

DOI: https://doi.org/10.20524/aog.2020.0489

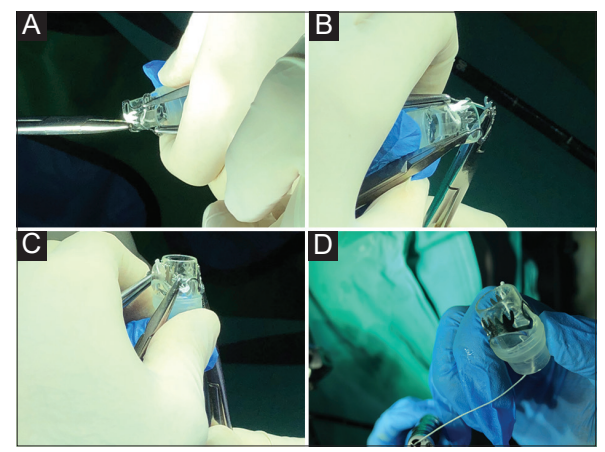

Figure 1 Reloading technique. (A) Fitting the clip in the cap and holding it stable with the 2 needle holders. (B) Pulling down the upper side. (C) Reopening of the clip. (D) Final placement
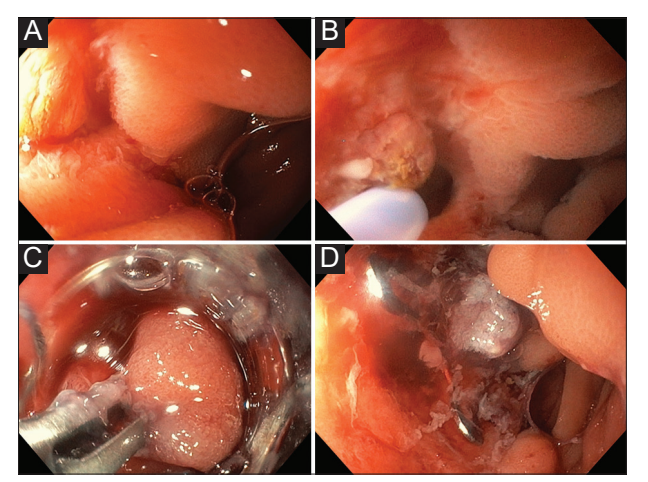

Figure 2 Endoscopic images. (A) Entry of the gastroscope into the area of the anastomosis. (B) Approaching the leakage. (C) Catching the tissue with the twin grasper. (D) Successful closure of the leakage with the over-the-scope clip

\section{Reference}

1. Mosquera-Klinger G, Torres-Rincón R, Jaime-Carvajal J. Endoscopic closure of gastrointestinal perforations and fistulas using the Ovesco over-the-scope clip system at a tertiary care hospital center. Rev Gastroenterol Mex 2019;84:263-266. 